\title{
OPEN SUsceptibility of bacterial endophthalmitis isolates to vancomycin, ceftazidime, and amikacin
}

\author{
Kuan-Jen Chen ${ }^{1,2} \bowtie$, Ming-Hui Sun ${ }^{1,2}$, Chiun-Ho Hou ${ }^{1,2}$, Hung-Chi Chen ${ }^{1,2}$, Yen-Po Chen ${ }^{1,2,3}$, \\ Nan-Kai Wang ${ }^{4}$, Laura Liu ${ }^{1,2}$, Wei-Chi Wu ${ }^{1,2}$, Hung-Da Chou ${ }^{1,2}$, Eugene Yu-Chuan Kang ${ }^{1,2}$ \& \\ Chi-Chun Lai ${ }^{1,2,5}$
}

Bacterial endophthalmitis is a rare intraocular infection, and prompt administration of intravitreal antibiotics is crucial for preventing severe vision loss. The retrospective study is to investigate the in vitro susceptibility to the antibiotics vancomycin, amikacin, and ceftazidime of bacterial endophthalmitis isolates in specimens at a tertiary referral center from January 1996 to April 2019 in Taiwan. Overall, 450 (49.9\%) isolates were Gram positive, 447 (49.6\%) were Gram negative, and $4(0.4 \%)$ were Gram variable. In Gram-positive isolates, coagulase-negative staphylococci were the most commonly cultured bacteria $(158,35.1 \%)$, followed by Streptococci $(100,22.2 \%)$, Enterococci $(75,16.7 \%)$, and Staphylococcus aureus (70, 15.6\%). In Gram-negative isolates, they were Klebsiella pneumoniae (166, 37.1\%) and Pseudomonas aeruginosa (131, 29.3\%). All Gram-positive organisms were susceptible to vancomycin, with the exception of one Enterococcus faecium isolate (1/450, $0.2 \%$ ). Of the Gram-negative isolates, $96.9 \%$ and $93.7 \%$ were susceptible to ceftazidime and amikacin, respectively. Nine isolates $(9 / 447,2.0 \%)$ were multidrug-resistant Gram-negative bacteria, comprising $K$. pneumoniae $(4 / 164,2.4 \%)$, Acinetobacter baumannii $(2 / 3,67 \%)$, and Stenotrophomonas maltophilia $(3 / 18,17 \%)$. In conclusion, in vitro susceptibility testing revealed that vancomycin remains the suitable antibiotic treatment for Gram-positive endophthalmitis. Ceftazidime and amikacin provide approximately the same degree of Gram-negative coverage. Multidrug-resistant bacterial endophthalmitis was uncommon.

Bacterial endophthalmitis is a rare intraocular infection that can occur following ocular surgery or trauma, as well as through the hematogenous spread of microorganisms from endogenous infection. Prompt administration of intravitreal antibiotics with or without pars plana vitrectomy is crucial for preventing severe vision loss. Both the bacterial spectrum and antibiotic susceptibility patterns in bacterial endophthalmitis isolates must be considered in the context of treatment. Vancomycin is a first-line drug for managing Gram-positive bacterial endophthalmitis, whereas ceftazidime or amikacin are typically used for Gram-negative coverage. Amid growing concern over the emergence of multidrug-resistant (MDR) bacteria, selecting antibiotics for infection treatment has become a critical issue. Common MDR organisms include (1) vancomycin-resistant enterococci; (2) methicillin-resistant S. aureus; (3) extended-spectrum $\beta$-lactamase (ESBL)-producing Gram-negative bacteria; (4) carbapenemaseproducing K. pneumoniae; and (5) MDR Gram-negative bacteria, such as K. pneumoniae, Enterobacter spp., Escherichia coli, A. baumannii, P. aeruginosa, and S. maltophilia. This group of Gram-positive and Gram-negative bacteria has been named the ESKAPE group (E. faecium, S. aureus, K. pneumoniae, A. baumannii, P. aeruginosa, and Enterobacter spp. $)^{1}$. Studies have been conducted on MDR bacterial endophthalmitis ${ }^{2-8}$. Although most isolates causing ocular infection are not caused by MDR bacteria, antibiotic susceptibility testing may reveal trends in antimicrobial susceptibility and resistance.

\footnotetext{
${ }^{1}$ Department of Ophthalmology, Chang Gung Memorial Hospital, Taoyüan, Taiwan. ${ }^{2}$ College of Medicine, Chang Gung University, Taoyüan, Taiwan. ${ }^{3}$ Department of Ophthalmology, Tucheng Municipal Hospital, New Taipei, Taiwan. ${ }^{4}$ Department of Ophthalmology, Edward S. Harkness Eye Institute, Columbia University, New York, NY, USA. ${ }^{5}$ Department of Ophthalmology, Chang Gung Memorial Hospital, Keelung, Taiwan. ${ }^{\square}$ email: cgr999@ gmail.com
} 
This study investigated the in vitro susceptibility of Gram-positive and Gram-negative bacterial endophthalmitis isolates collected over 23 years to vancomycin, ceftazidime, and amikacin. MDR pathogens were also examined.

\section{Methods}

The protocol of this retrospective, noncomparative laboratory case series was approved by the Institutional Review Board (IRB number: 201900614B0C601, 10 Aug 2019) of Chang Gung Memorial Hospital in Taiwan, and the requirement for written informed consent from the patients from whom the specimens were collected was waived. All clinical procedures were conducted according to the principles of the Declaration of Helsinki. The microbiological data of bacterial cultures isolated from culture-confirmed bacterial endophthalmitis at the Department of Microbiology of the participating hospital between January 1996 and April 2019 were reviewed. To protect the patients' privacy, the data were deidentified. Data on clinical presentations and visual outcomes were not part of the laboratory records.

The bacterial isolates are from intraocular samples (anterior chamber, vitreous, and/or vitrectomy specimens). All microbiology investigations were performed at Microbiology Department, Chang Gung Memorial Hospital, Taoyuan, Taiwan. Bacterial culture isolates were identified by conventional microbiological methods (January 1996 to December 2013) and matrix-assisted laser desorption/ionization time of flight mass spectrometry (MALDI-TOF-MS) (January 2014 to April 2019). Conventional microbiological methods included Gramstaining and biochemical tests. In MALDI-TOF-MS, automatic measurement of the spectrum and comparative analysis with reference spectra of bacteria were performed using an Ultraflextreme mass spectrometer and MALDI-Biotyper 3.0 software (Bruker Daltonics). The reliability of identification in the MALDI Biotyper system was expressed in points. A $\log ($ score $) \geq 2.0$ indicated identification to the species level. The isolates were tested for susceptibility to various antibiotics using the Kirby Bauer Disk diffusion method on Mueller Hinton blood agar. The Clinical and Laboratory Standards Institute (Wayne, PA) standards were used for interpretation and quality control for each corresponding year ${ }^{9}$. In vitro susceptibility of cultured bacterial organisms, the tested antibiotics mostly included either vancomycin for Gram-positive bacteria or ceftazidime and amikacin for Gramnegative bacteria. All data from the antibiotic susceptibility testing were further reviewed in the Gram-positive bacterial isolates resistant to vancomycin and in the Gram-negative bacteria resistant to either ceftazidime or amikacin. To be considered MDR, organisms must be resistant to antimicrobial drugs of three or more classes. Although methicillin-resistant $S$. aureus (MRSA) was susceptible to vancomycin, MRSA was not defined as an MDR organism in this study.

\section{Results}

A total of 901 bacterial endophthalmitis isolates were cultured over the 23-year study period. An overview of the isolates is provided in Table 1.

Organismal spectrum. Overall, 450 (49.9\%) of isolates were Gram positive, 447 (49.6\%) were Gram negative, and $4(0.4 \%)$ were Gram variable. In the $450 \mathrm{Gram}$-positive isolates, coagulase-negative staphylococci (CoNS) were the most commonly cultured bacterial organisms $(158,35.1 \%)$, followed by streptococci $(100$, $22.2 \%)$, enterococci $(75,16.7 \%)$, and $S$. aureus $(70,15.6 \%)$. In the 447 Gram-negative isolates, they were K. pneumoniae $(166,37.1 \%)$ and $P$. aeruginosa $(131,29.3 \%)$.

Susceptibility of Gram-positive isolates to vancomycin. Table 2 presents the susceptibility of the Gram-positive isolates to vancomycin. All Gram-positive bacteria, with the exception of one E. faecium isolate, were susceptible to vancomycin, including CoNS, S. aureus, Enterococcus faecalis, and streptococci (Table 2).

Susceptibility of Gram-negative isolates to amikacin and ceftazidime. Table 3 presents the susceptibility of the Gram-negative isolates to amikacin and ceftazidime. Overall, 96.9\% (413/426) were susceptible to ceftazidime and $93.7 \%$ (401/428) were susceptible to amikacin. Regarding the most commonly isolated Gram-negative organisms, $98 \%$ of the K. pneumoniae isolates were susceptible to both ceftazidime and amikacin. Of the $P$. aeruginosa isolates, $98 \%$ and $95 \%$ were susceptible to ceftazidime and amikacin, respectively. Of the S. maltophilia isolates, $8 \%$ and $46 \%$ were susceptible to ceftazidime and amikacin, respectively. All the Haemophilus influenzae, Serratia marcescens, Enterobacter spp., Citrobacter spp., and E. coli isolates were susceptible to ceftazidime and amikacin. Among them, MDR Gram-negative bacteria comprised K. pneumoniae (4/164, 2.4\%), A. baumannii $(2 / 3,67 \%)$, and S. maltophilia (3/18, 17\%). The S. maltophilia isolates exhibited high resistance to ceftazidime $(11 / 12,94 \%)$ and amikacin $(7 / 13,54 \%)$.

Susceptibility of Gram-variable isolates. Two Mycobacterium chelonae, one Mycobacterium abscessus, and one Nocardia spp. isolate comprised the Gram-variable isolates. Three Mycobacterium isolates were susceptible to amikacin but were not tested for susceptibility to ceftazidime. The Nocardia spp. isolate was not tested for susceptibility to amikacin and ceftazidime.

MDR organisms. Ten isolates (10/901, 1.1\%), including one E. faecium, four K. pneumoniae, two A. baumannii, and three $S$. maltophilia, were MDR organisms. Table 4 shows the antibiotic susceptibility testing results for multidrug resistance. The E. faecium isolate was susceptible to linezolid and tigecycline but resistant to teicoplanin. ESBL production was detected in the four K. pneumoniae isolates, of which only one was susceptible to amikacin. All four isolates were susceptible to carbapenems (imipenem, meropenem, or ertapenem). One of 


\begin{tabular}{|c|c|c|c|}
\hline Gram-positive & Genus or species & 450 & $49.9 \%$ \\
\hline \multirow{8}{*}{ Cocci } & \multicolumn{3}{|l|}{ Staphylococcus spp. } \\
\hline & CoNS & 158 & $17.5 \%$ \\
\hline & S. aureus & 70 & $7.8 \%$ \\
\hline & Streptococcus spp. & 100 & $11.1 \%$ \\
\hline & Enterococcus spp. & 75 & $8.3 \%$ \\
\hline & Aerococcus spp. & 1 & $0.1 \%$ \\
\hline & Micrococcus spp. & 3 & $0.3 \%$ \\
\hline & Rothia mucilaginosa & 1 & $0.1 \%$ \\
\hline \multirow{5}{*}{ Bacilli } & Corynebacterium spp. & 9 & $1.0 \%$ \\
\hline & Cutibacterium acnes & 10 & $1.1 \%$ \\
\hline & Paenibacillus spp. & 1 & $0.1 \%$ \\
\hline & Bacillus spp. & 18 & $2.0 \%$ \\
\hline & Clostridium spp. & 4 & $0.4 \%$ \\
\hline Gram-negative & Genus or species & 447 & $49.6 \%$ \\
\hline Cocci & Neisseria spp. & 4 & $0.4 \%$ \\
\hline Coccobacilli & Hemophilus spp. & 11 & $1.2 \%$ \\
\hline \multicolumn{4}{|l|}{ Bacilli } \\
\hline \multirow{8}{*}{ Enterobacteriaceae } & Klebsiella spp. & 166 & $18.4 \%$ \\
\hline & Citrobacter spp. & 8 & $0.9 \%$ \\
\hline & Enterobacter spp. & 16 & $1.8 \%$ \\
\hline & Morganella spp. & 2 & $0.2 \%$ \\
\hline & Proteus spp. & 12 & $1.3 \%$ \\
\hline & Salmonella spp. & 2 & $0.2 \%$ \\
\hline & Serratia spp. & 12 & $1.3 \%$ \\
\hline & Escherichia spp. & 13 & $1.4 \%$ \\
\hline \multirow{2}{*}{ Non-Enterobacteriaceae-fermentative } & Aeromonas spp. & 3 & $0.3 \%$ \\
\hline & Vibrio spp. & 1 & $0.1 \%$ \\
\hline \multirow{8}{*}{ Non-Enterobacteriaceae-nonfermentative } & Acinetobacter spp. & 5 & $0.6 \%$ \\
\hline & Burkholderia spp. & 4 & $0.4 \%$ \\
\hline & Flavobacterium spp. & 3 & $0.3 \%$ \\
\hline & Moraxella spp. & 4 & $0.4 \%$ \\
\hline & Pseudomonas spp. & 131 & $14.5 \%$ \\
\hline & Roseomonas spp. & 1 & $0.1 \%$ \\
\hline & Stenotrophomonas maltophilia & 18 & $2.0 \%$ \\
\hline & Others NF-GNB & 29 & $3.2 \%$ \\
\hline \multirow{2}{*}{ Anaerobic } & Bacteroides spp. & 1 & $0.1 \%$ \\
\hline & Prevotella spp. & 1 & $0.1 \%$ \\
\hline Gram variable bacilli & Genus or species & 4 & $0.4 \%$ \\
\hline Nontuberculous & Mycobacterium chelonae & 2 & $0.2 \%$ \\
\hline Mycobacteria & Mycobacterium abscesses & 1 & $0.1 \%$ \\
\hline Nocardia spp. & & 1 & $0.1 \%$ \\
\hline Total & & 901 & $100 \%$ \\
\hline
\end{tabular}

Table 1. Bacteria isolated from patients diagnosed with endophthalmitis. CoNS coagulase-negative Staphylococcus, NF-GNB non-fermentative-Gram-negative bacilli.

the A. baumannii isolates was susceptible to imipenem. The other was resistant to meropenem but susceptible to colistin and tigecycline. All three MDR S. maltophilia isolates were susceptible to amikacin but resistant to ceftazidime, trimethoprim-sulfamethoxazole, and fluoroquinolones, including levofloxacin and moxifloxacin.

\section{Discussion}

Understanding of the various organisms causing bacterial endophthalmitis is critical to the development of effective treatments. Case studies on bacterial endophthalmitis have reported variability in the microbiological spectrum of causative pathogens according to the types of endophthalmitis and to the regions and countries where the studies were conducted. Bacterial endophthalmitis is caused by Gram-positive and Gram-negative organisms, as well as some Gram-variable organisms. Empirically supported antibiotic treatments for infectious endophthalmitis are developed on the basis of likely causative organisms and their susceptibility patterns. In this study, the proportions of Gram-positive and Gram-negative bacteria ( $49.9 \%$ and $49.6 \%$, respectively) 


\begin{tabular}{|c|c|c|c|}
\hline \multirow[b]{2}{*}{ Gram-positive bacteria } & & \multicolumn{2}{|c|}{ Vancomycin } \\
\hline & & Number & $\%$ \\
\hline \multicolumn{4}{|l|}{ Staphylococcus spp. } \\
\hline CoNS & 158 & $158 / 158$ & $100 \%$ \\
\hline S. aureus & 70 & $70 / 70$ & $100 \%$ \\
\hline Streptococcus spp. & 100 & $100 / 100$ & $100 \%$ \\
\hline Enterococcus spp. & 75 & $74 / 75$ & $98.7 \%$ \\
\hline Aerococcus spp. & 1 & $1 / 1$ & $100 \%$ \\
\hline Micrococcus spp. & 3 & $3 / 3$ & $100 \%$ \\
\hline Rothia mucilaginosa & 1 & $1 / 1$ & $100 \%$ \\
\hline Corynebacterium spp. & 9 & $9 / 9$ & $100 \%$ \\
\hline Cutibacterium acnes & 10 & $10 / 10$ & $100 \%$ \\
\hline Paenibacillus spp. & 1 & $1 / 1$ & $100 \%$ \\
\hline Bacillus spp. & 18 & $18 / 18$ & $100 \%$ \\
\hline \multirow{2}{*}{ Clostridium spp. } & 4 & $4 / 4$ & $100 \%$ \\
\hline & 450 & $449 / 450$ & $99.8 \%$ \\
\hline
\end{tabular}

Table 2. Vancomycin susceptibility testing in Gram positive bacteria. CoNS coagulase-negative Staphylococcus.

were approximately equal, with Gram-variable bacteria accounting for a considerably lower proportion (0.4\%). Exogenous bacterial endophthalmitis mostly arises form Gram-positives because Gram-negatives are not common on the eyelid margins. This is partially attributable to the large number of reported cases of endogenous $K$. pneumoniae endophthalmitis and P. aeruginosa keratitis-related endophthalmitis in Taiwan ${ }^{4,6,10-12}$. Our data differ from those of other published reviews of endophthalmitis isolates (Table 5) ${ }^{13-19}$. Gram-positive bacteria account for most bacterial isolates of endophthalmitis in studies conducted in the United States, Canada, and Austria, whereas Gram-negative bacteria are predominant in India ${ }^{13-19}$. The higher proportion of Gram-negative isolates observed in one study ${ }^{17}$ from India may be explained by trauma and environmental factors. In this study, $1.1 \%$ (10/901) of the MDR bacterial isolates included one Gram-positive and nine Gram-negative bacterial organisms.

As mentioned, the most commonly cultured Gram-positive isolates were CoNS, followed by Streptococcus spp., Enterococcus spp., and S. aureus. These results are consistent with those from previous studies ${ }^{13-17}$. However, a higher proportion of Enterococcus spp., especially E. faecalis, was noted in this study, and it resulted from more E. faecalis endophthalmitis cases in Taiwan ${ }^{20,21}$. Gram-positive vancomycin-resistant endophthalmitis has become a critical clinical issue worldwide; this is indicated by the fact that Enterococcus spp. and Staphylococcus spp. were the most commonly reported organisms ${ }^{7}$. The rate of Gram-positive vancomycin resistance in the present study was $0.2 \%$. All Staphylococcus spp. isolates were susceptible to vancomycin, but 1 of the 75 (1.3\%) Enterococcus isolates, E. faecium, was resistant to vancomycin. Several case reports have examined vancomycinresistant enterococcal endophthalmitis ${ }^{22-24}$. E. faecalis was less commonly resistant to vancomycin. In other case reports of endophthalmitis, E. casseliflavus ${ }^{25}$ and $E$. faecium ${ }^{2}$ exhibited higher rates of resistance to vancomycin. In a literature review ${ }^{7}$ of 27 types of vancomycin-resistant Gram-positive bacteria, Enterococcus spp., CoNS, $S$. aureus, and Bacillus spp. were the most common organisms.

In this study, $3.1 \%$ and $6.3 \%$ of the Gram-negative bacteria was resistant to ceftazidime and amikacin, respectively. In these resistant isolates, most Gram-negative bacteria were resistant to either ceftazidime or amikacin. However, five isolates $(5 / 447,1.1 \%)$ were resistant to both ceftazidime and amikacin. Apart from four K. pneumoniae isolates, most bacteria in the Enterobacteriaces family were susceptible to amikacin and ceftazidime. By comparison, nonfermenting Gram-negative bacteria not belonging to the Enterobacteriaces family were more likely to be resistant to ceftazidime and amikacin. P. aeruginosa had a comparable degree of susceptibility to ceftazidime (98\%) and amikacin (95\%). By contrast, the susceptibility of S. maltophilia to ceftazidime and amikacin differed substantially ( $8 \%$ and $46 \%$, respectively).

Among the MDR bacteria, E. faecium was susceptible to linezolid and tigecycline. The nine MDR Gramnegative bacterial isolates comprised K. pneumoniae (4/164, 2.4\%), A. baumannii (2/3,67\%), and S. maltophilia $(3 / 18,17 \%)$. The ceftazidime resistance of K. pneumoniae, a member of the Enterobacteriaceae family, was indicated by the presence of ESBL production. The ESBL-producing Gram-negative bacteria were resistant to all third-generation cephalosporins. Four MDR K. pneumoniae isolates were susceptible to carbapenems (imipenem, meropenem, or ertapenem). One of the A. baumannii isolates was susceptible to carbapenem, and the other was susceptible to colistin and tigecycline. All three MDR S. maltophilia isolates were susceptible to amikacin but resistant to ceftazidime, trimethoprim-sulfamethoxazole, and fluoroquinolones, including levofloxacin and moxifloxacin. In a case report of carbapenemase-producing K. pneumoniae endophthalmitis by Zhou et al. ${ }^{8}$, the $K$. pneumoniae isolate was resistant to amikacin, ceftazidime, and carbapenems but susceptible to polymyxin $\mathrm{E}$ and tigecycline. Roy et al. ${ }^{26}$ noted that four (100\%) A. baumannii isolates were resistant to ceftazidime and one (25\%) was resistant to amikacin. All four of those A. baumannii isolates were susceptible to ciprofloxacin ${ }^{26}$. Although S. maltophilia was typically susceptible to trimethoprim-sulfamethoxazole, levofloxacin, and moxifloxacin, Ji et al. ${ }^{5}$ reported on 14 patients with MDR S. maltophilia postcataract endophthalmitis. All eight $S$. 


\begin{tabular}{|c|c|c|c|c|c|}
\hline Gram-negative & Genus & Species & No. & Amikacin & Ceftazidime \\
\hline Cocci & Neisseria & & 4 & $1 / 1(100 \%)$ & $1 / 1(100 \%)$ \\
\hline Coccobacilli & Hemophilus & H. influenzae & 11 & $11 / 11(100 \%)$ & $11 / 11(100 \%)$ \\
\hline \multicolumn{6}{|l|}{ Bacilli } \\
\hline \multirow{14}{*}{ Enterobacteriaceae } & \multirow{2}{*}{ Klebsiella } & K. pneumoniae & 164 & $161 / 164(98 \%)$ & $160 / 164(98 \%)$ \\
\hline & & K. oxytoca & 2 & $2 / 2(100 \%)$ & $2 / 2(100 \%)$ \\
\hline & \multirow{2}{*}{ Citrobacter } & C. freundii & 4 & $4 / 4(100 \%)$ & $4 / 4(100 \%)$ \\
\hline & & C. koseri & 4 & $4 / 4(100 \%)$ & $4 / 4(100 \%)$ \\
\hline & \multirow{4}{*}{ Enterobacter } & E. aerogenes & 1 & $1 / 1(100 \%)$ & $1 / 1(100 \%)$ \\
\hline & & E. agglomerans & 2 & $2 / 2(100 \%)$ & $2 / 2(100 \%)$ \\
\hline & & E. cloacae & 11 & $11 / 11(100 \%)$ & $11 / 11(100 \%)$ \\
\hline & & E. gergoviae & 2 & $2 / 2(100 \%)$ & $2 / 2(100 \%)$ \\
\hline & Morganella & M. morganii & 2 & $2 / 2(100 \%)$ & $2 / 2(100 \%)$ \\
\hline & \multirow{2}{*}{ Proteus } & P. mirabilis & 11 & $11 / 11(100 \%)$ & $11 / 11(100 \%)$ \\
\hline & & P. vulgaris & 1 & $1 / 1(100 \%)$ & $1 / 1(100 \%)$ \\
\hline & Salmonella & & 2 & NT & $2 / 2(100 \%)$ \\
\hline & Serratia & S. marcescens & 12 & $12 / 12(100 \%)$ & $12 / 12(100 \%)$ \\
\hline & Escherichia & E. coli & 13 & $13 / 13(100 \%)$ & $13 / 13(100 \%)$ \\
\hline \multirow{2}{*}{ Non-Enterobacteriaceae-fermentative } & Aeromonas & A. hydrophilia & 3 & $3 / 3(100 \%)$ & $3 / 3(100 \%)$ \\
\hline & Vibrio & V.parahaemolyticus & 1 & NT & $1 / 1(100 \%)$ \\
\hline \multirow{16}{*}{ Non-Enterobacteriaceae-nonfermentative } & \multirow{3}{*}{ Acinetobacter } & A. baumannii & 3 & $1 / 3(33 \%)$ & $1 / 3(33 \%)$ \\
\hline & & A. lwoffii & 1 & $1 / 1(100 \%)$ & $1 / 1(100 \%)$ \\
\hline & & A. pittii & 1 & $1 / 1(100 \%)$ & $1 / 1(100 \%)$ \\
\hline & \multirow{2}{*}{ Burkholderia } & B. pseudomallei & 1 & $0 / 1(0 \%)$ & $1 / 1(100 \%)$ \\
\hline & & B. cepacia complex & 3 & $0 / 1(0 \%)$ & $3 / 3(100 \%)$ \\
\hline & Flavobacterium & & 3 & $3 / 3(100 \%)$ & $3 / 3(100 \%)$ \\
\hline & \multirow{2}{*}{ Moraxella } & M. catarrhalis & 3 & NT & $3 / 3(100 \%)$ \\
\hline & & M. nonliquefaciens & 1 & NT & $1 / 1(100 \%)$ \\
\hline & \multirow{5}{*}{ Pseudomonas } & P. aeruginosa & 123 & $117 / 123(95 \%)$ & $121 / 123(98 \%)$ \\
\hline & & P. fluorescens & 2 & $2 / 2(100 \%)$ & $2 / 2(100 \%)$ \\
\hline & & P. luteola & 1 & $1 / 1(100 \%)$ & $1 / 1(100 \%)$ \\
\hline & & P. stutzeri & 2 & $2 / 2(100 \%)$ & $2 / 2(100 \%)$ \\
\hline & & Non-identified & 3 & NT & NT \\
\hline & Roseomonas & & 1 & $1 / 1(100 \%)$ & $0 / 1(0 \%)$ \\
\hline & Stenotrophomonas & S. maltophilia & 18 & $6 / 13(46 \%)$ & $1 / 12(8 \%)$ \\
\hline & Others NF-GNB & & 29 & $22 / 29(76 \%)$ & $25 / 29(86 \%)$ \\
\hline \multirow{3}{*}{ Anaerobic } & Bacteroides & & 1 & NT & NT \\
\hline & \multirow{2}{*}{ Prevotella } & & 1 & NT & NT \\
\hline & & & 447 & $401 / 428(93.7 \%)$ & $413 / 426(96.9 \%)$ \\
\hline
\end{tabular}

Table 3. Susceptibility of Gram-negative bacterial isolates to ceftazidime and amikacin. NFGNB nonfermenting Gram-negative bacilli, NT not tested.

maltophilia isolates were resistant to amikacin, imipenem, and ciprofloxacin but not to levofloxacin ${ }^{5}$. Five isolates were resistant to ceftazidime ${ }^{5}$.

Most bacteria are typically susceptible to vancomycin, ceftazidime, and amikacin at a concentration of $\leq 10 \mu \mathrm{g} /$ $\mathrm{mL}$. Interpretive standards for dilution susceptibility testing from the US Clinical and Laboratory Standards Institute indicate that the minimal inhibitory concentrations of vancomycin, ceftazidime, and amikacin are $\leq 4, \leq 8$, and $\leq 6 \mu \mathrm{g} / \mathrm{mL}$, respectively ${ }^{27}$. The resistance concentrations of vancomycin, ceftazidime, and amikacin are $\geq 32, \geq 32$, and $\geq 64 \mu \mathrm{g} / \mathrm{mL}$, respectivel ${ }^{27}$. Intravitreal antibiotic injection is the principal treatment for infectious endophthalmitis. The susceptibility standards are based on serum standards used for systemic therapy from the Clinical and Laboratory Standards Institute. However, there are no standards for intravitreal therapy, and resistance is probably over-reported using the serum standards. In vitro resistance patterns may not be identical with in vivo susceptibility, and routinely administered intravitreal antibiotics typically deliver intraocular antibiotic concentrations that are considerably higher than the minimal inhibitory concentrations of most organisms. When patients' conditions do not stabilize or improve after initial intravitreal antibiotic injection, additional injections of alternative antibiotics should be considered according to results in vitro susceptibility testing.

This study has some limitations. First, this was a single-center retrospective case series. Second, some Gramnegative isolates were not routinely tested at our hospital for susceptibility to amikacin and ceftazidime. Third, 


\begin{tabular}{|c|c|c|c|c|c|c|c|c|c|c|}
\hline \multirow{3}{*}{$\begin{array}{l}\text { Antibiotics } \\
\text { No. of isolates }\end{array}$} & \multicolumn{10}{|l|}{ Organisms } \\
\hline & \multirow{2}{*}{\begin{tabular}{|l|} 
Enterococcus faecium \\
1
\end{tabular}} & \multicolumn{4}{|c|}{ Klebsiella pneumoniae } & \multicolumn{2}{|c|}{$\begin{array}{l}\text { Acinetobacter } \\
\text { baumannii }\end{array}$} & \multicolumn{3}{|c|}{$\begin{array}{l}\text { Stenotrophomonas } \\
\text { maltophilia }\end{array}$} \\
\hline & & 1 & 2 & 3 & 4 & 1 & 2 & 1 & 2 & 3 \\
\hline Penicillin & $\mathrm{R}$ & & & & & & & & & \\
\hline Ampicillin & $\mathrm{R}$ & $\mathrm{R}$ & $\mathrm{R}$ & $\mathrm{R}$ & & $\mathrm{R}$ & & & & \\
\hline Amoxicillin-CLA & & & & & S & & & & & \\
\hline Ampicillin-SUL & & & & & & & $\mathrm{R}$ & & & \\
\hline Piperacillin & & $\mathrm{R}$ & $\mathrm{R}$ & S & $\mathrm{R}$ & $\mathrm{R}$ & $\mathrm{R}$ & & & \\
\hline Piperacillin-TZP & & & S & & S & & $\mathrm{R}$ & & & \\
\hline Vancomycin & $\mathrm{R}$ & & & & & & & & & \\
\hline Teicoplanin & $\mathrm{R}$ & & & & & & & & & \\
\hline Linezolid & S & & & & & & & & & \\
\hline Tigecycline & S & & & & & & $S$ & $\mathrm{R}$ & $\mathrm{R}$ & I \\
\hline Gentamicin & & $\mathrm{R}$ & $\mathrm{R}$ & $\mathrm{R}$ & $\mathrm{R}$ & $\mathrm{R}$ & $\mathrm{R}$ & & & \\
\hline High level GM & $\mathrm{R}$ & & & & & & & & & \\
\hline Amikacin & & $\mathrm{R}$ & $\mathrm{R}$ & $\mathrm{R}$ & $\mathrm{S}$ & $\mathrm{R}$ & $\mathrm{R}$ & $\mathrm{S}$ & $\mathrm{S}$ & $S$ \\
\hline Cefazolin & & $\mathrm{R}$ & $\mathrm{R}$ & $\mathrm{R}$ & $\mathrm{R}$ & $\mathrm{R}$ & & & & \\
\hline Cefuroxime & & $\mathrm{R}$ & $\mathrm{R}$ & $\mathrm{R}$ & $\mathrm{R}$ & $\mathrm{R}$ & & $\mathrm{R}$ & $\mathrm{R}$ & $\mathrm{R}$ \\
\hline Ceftriaxone & & $\mathrm{R}$ & $\mathrm{R}$ & $\mathrm{R}$ & $\mathrm{R}$ & $\mathrm{R}$ & $\mathrm{R}$ & & & \\
\hline Ceftazidime & & $\mathrm{R}$ & $\mathrm{R}$ & $\mathrm{R}$ & $\mathrm{R}$ & $\mathrm{R}$ & $\mathrm{R}$ & $\mathrm{R}$ & $\mathrm{R}$ & $\mathrm{R}$ \\
\hline Floxomef & & $\mathrm{R}$ & $\mathrm{R}$ & S & S & $\mathrm{R}$ & & & & \\
\hline Cefepime & & & & & & & $\mathrm{R}$ & & & \\
\hline Aztreonam & & $\mathrm{R}$ & $\mathrm{R}$ & $\mathrm{R}$ & $\mathrm{R}$ & $\mathrm{R}$ & & & & \\
\hline TMP-SMX & & $\mathrm{R}$ & $\mathrm{R}$ & $\mathrm{R}$ & & $\mathrm{R}$ & & $\mathrm{R}$ & $\mathrm{R}$ & $\mathrm{R}$ \\
\hline Ciprofloxacin & & S & $\mathrm{R}$ & S & $S$ & $\mathrm{R}$ & $\mathrm{R}$ & & & \\
\hline Levofloxacin & & & & & & & & $\mathrm{R}$ & $\mathrm{R}$ & $\mathrm{R}$ \\
\hline Moxifloxacin & & & & & & & & $\mathrm{R}$ & $\mathrm{R}$ & $\mathrm{R}$ \\
\hline Imipenem & & S & & S & & S & & & & \\
\hline Meropenem & & & S & & & & $\mathrm{R}$ & & & \\
\hline Ertapenem & & & & & $S$ & & & & & \\
\hline Colistin & & & & & & & S & & & \\
\hline
\end{tabular}

Table 4. Antibiotic susceptibility testing in multidrug-resistant organisms. CLA clavulanic acid, $I$ intermediate, $S$ susceptible, $S U L$ sulbactam, $R$ resistant, TMP-SXT trimethoprim-sulfamethoxazole, TZP tazobactam.

\begin{tabular}{|c|c|c|c|c|c|c|c|c|}
\hline & $\mathrm{UPMC}^{19}$ & NYEEI $^{13}$ & BPEI $^{15}$ & Toronto $^{18}$ & Hyderabad $^{17}$ & Hyderabad $^{14}$ & Queensland $^{16}$ & CGMH $^{*}$ \\
\hline & USA & USA & USA & Canada & India & India & Australia & Taiwan \\
\hline Study period & 1993-2015 & $1987-2011$ & $2002-2011$ & 2000-2009 & 1991-2015 & $2010-2013$ & $1998-2013$ & 1996-2019 \\
\hline No. of isolates & 665 & 943 & 375 & 265 & 2840 & 196 & 193 & 901 \\
\hline $\begin{array}{l}\text { Gram-positive } \\
\text { isolates }\end{array}$ & $92.9 \%$ & $89.2 \%$ & $87.2 \%$ & $90.2 \%$ & $68.7 \%$ & $37.2 \%$ & $84.5 \%$ & $49.9 \%$ \\
\hline $\begin{array}{l}\text { Susceptibility to } \\
\text { vancomycin }\end{array}$ & $99.7 \%$ & $99.7 \%$ & $100.0 \%$ & $99.6 \%$ & $96 \%$ & $100.0 \%$ & $100.0 \%$ & $99.8 \%$ \\
\hline $\begin{array}{l}\text { Gram-negative } \\
\text { isolates }\end{array}$ & $7.1 \%$ & $10.8 \%$ & $12.8 \%$ & $9.8 \%$ & $32.3 \%$ & $62.8 \%$ & $15.5 \%$ & $49.6 \%$ \\
\hline $\begin{array}{l}\text { Susceptibility to } \\
\text { amikacin }\end{array}$ & $95.7 \%$ & $92.9 \%$ & N/A & N/A & $64-67 \%{ }^{\mathrm{a}}$ & $87.0 \%$ & $100.0 \%$ & $93.7 \%$ \\
\hline $\begin{array}{l}\text { Susceptibility to } \\
\text { ceftazidime }\end{array}$ & $93.6 \%$ & $91.5 \%$ & $100.0 \%$ & $100.0 \%$ & $69-38 \%^{\mathrm{a}}$ & $82.0 \%$ & $100.0 \%$ & $96.9 \%$ \\
\hline
\end{tabular}

Table 5. Review and comparison of bacterial endophthalmitis isolates. BPEI Bascom Palmer Eye Institute, CGMH Chang Gung Memorial Hospital, N/A not available, NYEEI New York Eye and Ear Infirmary, UPMC

University of Pittsburgh Medical Center. ${ }^{*}$ Including 4 Gram-variable isolates. ${ }^{a} 2005 \rightarrow 2015$. 
we did not retrieve accurate, detailed information on various types of endophthalmitis and visual outcomes, which may interest readers. Fourth, we did not analyze trends in the patterns of antibiotic susceptibility given the relatively small number of annual cases. Finally, we did not further analyze the MDR bacteria for specific resistance genes. Nevertheless, our findings contribute to the literature in providing a thorough analysis of the antimicrobial susceptibility of bacterial isolates from all patients diagnosed as having bacterial endophthalmitis over 23 years at a tertiary medical center.

In conclusion, the most commonly detected organisms in the Gram-positive and Gram-negative bacteria were CoNS and K. pneumoniae, respectively. MDR bacterial endophthalmitis was not common. Vancomycin remains the antibiotic of choice for the treatment of Gram-positive endophthalmitis. Amikacin and ceftazidime appear to provide approximately equal Gram-negative coverage.

Received: 2 July 2021; Accepted: 27 July 2021

Published online: 05 August 2021

\section{References}

1. Boucher, H. W. et al. Bad bugs, no drugs: No ESKAPE! An update from the Infectious Diseases Society of America. Clin. Infect. Dis. Off. Publ. Infect. Dis. Soc. Am. 48, 1-12. https://doi.org/10.1086/595011 (2009).

2. Bains, H. S., Weinberg, D. V., Feder, R. S. \& Noskin, G. A. Postoperative vancomycin-resistant Enterococcus faecium endophthalmitis. Arch. Ophthalmol. (Chicago Ill. 1960) 125, 1292-1293. https://doi.org/10.1001/archopht.125.9.1292 (2007).

3. Chen, K. J. et al. Endophthalmitis caused by Acinetobacter baumannii: Report of two cases. J. Clin. Microbiol. 46, 1148-1150. https://doi.org/10.1128/jcm.01604-07 (2008).

4. Chen, K. J. et al. Endogenous Klebsiella endophthalmitis associated with Klebsiella pneumoniae pneumonia. Ocul. Immunol. Inflamm. 17, 153-159. https://doi.org/10.1080/09273940902752250 (2009).

5. Ji, Y. et al. Post-cataract endophthalmitis caused by multidrug-resistant Stenotrophomonas maltophilia: Clinical features and risk factors. BMC Ophthalmol. 15, 14. https://doi.org/10.1186/1471-2415-15-14 (2015).

6. Li, Y. H. et al. Infectious sources, prognostic factors, and visual outcomes of endogenous Klebsiella pneumoniae endophthalmitis. Ophthalmol. Retina 2, 771-778. https://doi.org/10.1016/j.oret.2017.11.013 (2018).

7. Relhan, N. et al. Endophthalmitis caused by Gram-positive organisms with reduced vancomycin susceptibility: Literature review and options for treatment. Br. J. Ophthalmol. 100, 446-452. https://doi.org/10.1136/bjophthalmol-2015-307722 (2016).

8. Zhou, Y., Wang, X., Shen, J., Lu, Z. \& Liu, Y. Endogenous Endophthalmitis caused by Carbapenem-resistant Hypervirulent Klebsiella Pneumoniae: A case report and literature review. Ocul. Immunol. Inflamm. 27, 1099-1104. https://doi.org/10.1080/09273948.2018. 1502786 (2019).

9. Clinical and Laboratory Standards Institute. Performance Standards for Antimicrobial Disk Susceptibility Tests; Approved Standard, 12th edn. M02-A13. (Clinical and Laboratory Standards Institute).

10. Chen, Y. H. et al. Prognostic factors and visual outcomes of pyogenic liver abscess-related endogenous Klebsiella pneumoniae endophthalmitis: A 20-year retrospective review. Sci. Rep. 9, 1071. https://doi.org/10.1038/s41598-018-37643-y (2019).

11. Chen, K. J. et al. Endophthalmitis caused by Pseudomonas aeruginosa in Taiwan. Retina (Phila. Pa.) 31, 1193-1198. https://doi. org/10.1097/IAE.0b013e3181fbce5c (2011).

12. Chen, K. J., Wu, W. C., Sun, M. H., Lai, C. C. \& Chen, T. L. Pseudomonas endophthalmitis. Ophthalmology 117, 1657-1658. https:// doi.org/10.1016/j.ophtha.2010.04.011 (2010) (author reply 1658-1659).

13. Gentile, R. C. et al. Microbiological spectrum and antibiotic sensitivity in endophthalmitis: A 25-year review. Ophthalmology 121, 1634-1642. https://doi.org/10.1016/j.ophtha.2014.02.001 (2014).

14. Reddy, A. K. et al. Susceptibility of bacterial isolates to vancomycin and ceftazidime from patients with endophthalmitis: Is there a need to change the empirical therapy in suspected bacterial endophthalmitis?. Int. Ophthalmol. 35, 37-42. https://doi.org/10. 1007/s10792-014-0015-9 (2015).

15. Schimel, A. M., Miller, D. \& Flynn, H. W. Jr. Endophthalmitis isolates and antibiotic susceptibilities: A 10-year review of cultureproven cases. Am. J. Ophthalmol. 156, 50-52.e51. https://doi.org/10.1016/j.ajo.2013.01.027 (2013).

16. Moloney, T. P. \& Park, J. Microbiological isolates and antibiotic sensitivities in culture-proven endophthalmitis: A 15 -year review. Br. J. Ophthalmol. 98, 1492-1497. https://doi.org/10.1136/bjophthalmol-2014-305030 (2014).

17. Joseph, J. et al. Trends in microbiological spectrum of endophthalmitis at a single tertiary care ophthalmic hospital in India: A review of 25 years. Eye (Lond.) 33, 1090-1095. https://doi.org/10.1038/s41433-019-0380-8 (2019).

18. Assaad, D. et al. Bacterial endophthalmitis: 10-year review of the culture and sensitivity patterns of bacterial isolates. Can. J. Ophthalmol. J. Can. D'ophtalmol. 50, 433-437. https://doi.org/10.1016/j.jcjo.2015.07.013 (2015).

19. Kodati, S., Eller, A. W. \& Kowalski, R. P. The susceptibility of bacterial endophthalmitis isolates to vancomycin, ceftazidime, and amikacin: A 23 year-review. Ophthalmol. Retina 1, 206-209. https://doi.org/10.1016/j.oret.2016.11.010 (2017).

20. Chen, K. J. et al. Postcataract endophthalmitis caused by Enterococcus faecalis. Ocul. Immunol. Inflamm. 17, 364-369. https://doi. org/10.3109/09273940903105110 (2009).

21. Chen, K. J. et al. Enterococcus faecalis endophthalmitis: Clinical settings, antibiotic susceptibility, and management outcomes. Microorganisms https://doi.org/10.3390/microorganisms9050918 (2021).

22. Dave, V. P. et al. Enterococcus endophthalmitis: Clinical settings, antimicrobial susceptibility, and management outcomes. Retina (Phila. Pa.) 40, 898-902. https://doi.org/10.1097/iae.0000000000002462 (2020).

23. Rishi, E., Rishi, P., Nandi, K., Shroff, D. \& Therese, K. L. Endophthalmitis caused by Enterococcus faecalis: A case series. Retina (Phila. Pa.) 29, 214-217. https://doi.org/10.1097/IAE.0b013e31818eccc7 (2009).

24. Tang, C. W., Cheng, C. K. \& Lee, T. S. Community-acquired bleb-related endophthalmitis caused by vancomycin-resistant enterococci. Can. J. Ophthalmol. J. Can. D'ophtalmol. 42, 477-478 (2007).

25. Low, J. R., Teoh, C. S., Chien, J. M. \& Huang, E. H. Enterococcus casseliflavus endophthalmitis due to metallic intraocular foreign body. Eye (Lond.) 29, 840-841. https://doi.org/10.1038/eye.2014.332 (2015).

26. Roy, R. et al. Endophthalmitis caused by Acinetobacter baumanni: A case series. Eye (Lond.) 27, 450-452. https://doi.org/10.1038/ eye.2012.277 (2013).

27. Performance Standards for Antimicrobial Susceptibility Testing. Clinical and Laboratory Standards Institute (CLSI). M100 28th edition (2018).

\section{Acknowledgements}

The authors wish to acknowledge the support of the Taiwan Ministry of Science and Technology MOST 106-2314-B-182A-041, and Chang Gung Memorial Hospital CMRPG3F1243. 


\section{Author contributions}

K.-J.C. designed the study, performed statistical analysis, wrote the manuscript, and approved the final manuscript for publication; M.-H.S. collected and analyzed the data; H.-C.H., H.-C.C., Y.-P.C., N.-K.W., L.L., W.C.W., H.-D.C., E.Y.-C.K. and C.-C.L. all provided patients' data and assisted in data collection. All authors have reviewed the manuscript.

\section{Competing interests}

The authors declare no competing interests.

\section{Additional information}

Correspondence and requests for materials should be addressed to K.-J.C.

Reprints and permissions information is available at www.nature.com/reprints.

Publisher's note Springer Nature remains neutral with regard to jurisdictional claims in published maps and institutional affiliations.

(c) (i) Open Access This article is licensed under a Creative Commons Attribution 4.0 International License, which permits use, sharing, adaptation, distribution and reproduction in any medium or format, as long as you give appropriate credit to the original author(s) and the source, provide a link to the Creative Commons licence, and indicate if changes were made. The images or other third party material in this article are included in the article's Creative Commons licence, unless indicated otherwise in a credit line to the material. If material is not included in the article's Creative Commons licence and your intended use is not permitted by statutory regulation or exceeds the permitted use, you will need to obtain permission directly from the copyright holder. To view a copy of this licence, visit http://creativecommons.org/licenses/by/4.0/.

(C) The Author(s) 2021 